\title{
Multisensory Integration in Dynamical Behaviors: Maximum Likelihood Estimation across Bimanual Skill Learning
}

\author{
Renaud Ronsse, ${ }^{1}$ R. Chris Miall, ${ }^{2}$ and Stephan P. Swinnen ${ }^{1}$ \\ ${ }^{1}$ Motor Control Laboratory, Department of Biomedical Kinesiology, Katholieke Universiteit Leuven, B-3001 Heverlee, Belgium, and ${ }^{2}$ School of Psychology, \\ University of Birmingham, Edgbaston, Birmingham B15 2TT, United Kingdom
}

Optimal integration of different sensory modalities weights each modality as a function of its degree of certainty (maximum likelihood). Humans rely on near-optimal integration in decision-making tasks (involving e.g., auditory, visual, and/or tactile afferents), and some support for these processes has also been provided for discrete sensorimotor tasks. Here, we tested optimal integration during the continuous execution of a motor task, using a cyclical bimanual coordination pattern in which feedback was provided by means of proprioception and augmented visual feedback (AVF, the position of both wrists being displayed as the orthogonal coordinates of a single cursor). Assuming maximum likelihood integration, the following predictions were addressed: (1) the coordination variability with both AVF and proprioception available is smaller than with only one of the two modalities, and should reach an optimal level; (2) if the AVF is artificially corrupted by noise, variability should increase but saturate toward the level without AVF; (3) if the AVF is imperceptibly phase shifted, the stabilized pattern should be partly adapted to compensate for this phase shift, whereby the amount of compensation reflects the weight assigned to AVF in the computation of the integrated signal. Whereas performance variability gradually decreased over $5 \mathrm{~d}$ of practice, we showed that these model-based predictions were already observed on the first day. This suggests not only that the performer integrated proprioceptive feedback and AVF online during task execution by tending to optimize the signal statistics, but also that this occurred before reaching an asymptotic performance level.

\section{Introduction}

The integration of different sensory modalities occurs at various nodes in the human brain (Holmes and Spence, 2005; Macaluso, 2006; Driver and Noesselt, 2008; Stein and Stanford, 2008). This process is thought to be important to merge multiple, often redundant, sources of information into a common representation about the outside world. It has been studied across a broad range of behaviors, and can be modeled assuming optimality, i.e., maximum likelihood estimation (MLE): different signal sources are weighted by the inverse of their variance, greater weight being given to the more reliable source (Clark and Yuille, 1990; Hillis et al., 2002). This is seen in the combination of different sensory modalities (van Beers et al., 1999; Ernst and Banks, 2002; Sober and Sabes, 2003; Alais and Burr, 2004; Helbig and Ernst, 2007; Körding et al., 2007), or in the inference from prior knowledge about stimulus distribution and actual sensory input (Bayesian statistics) (Kersten and Yuille, 2003; Körding and Wolpert, 2004, 2006, 2007; Tassinari et al., 2006; Vaziri et al., 2006). Most of these studies focused on the statistics of decision-making pro-

\footnotetext{
Received Dec. 2, 2008; revised March 23, 2009; accepted March 27, 2009.

Support for this study was provided through a grant from the Research Fund Katholieke Universiteit Leuven (0T/07/073) and the Flanders Fund for Scientific Research (G.0241.05, G.0292.05, G.0593.08). This work was also supported by Grant P6/29 from the Interuniversity Attraction Poles program of the Belgian federal government. R.R. was funded by the Francqui Foundation. R.C.M. was funded by the Wellcome Trust.

Correspondence should be addressed to Stephan P. Swinnen, Motor Control Laboratory, Department of Biomedical Kinesiology, Katholieke Universiteit Leuven, Tervuursevest 101 — bus 1501, B-3001 Heverlee, Belgium. E-mail: stephan.swinnen@faber.kuleuven.be.

DOI:10.1523/JNEUROSCI.5734-08.2009

Copyright $\odot 2009$ Society for Neuroscience $\quad$ 0270-6474/09/298419-10\$15.00/0
}

cesses (asking, e.g., "Are the visual and auditory sources congruent or incongruent?") or on the distribution of endpoint discrete reaching movements, i.e., movements with clearly defined beginning points and endpoints (Magill, 2006; Hogan and Sternad, 2007).

Here, we focused on optimal multisensory integration in the context of coordinated movement control. Human subjects were instructed to continuously stabilize a complex bimanual pattern: cyclical movement of the two wrists, one being a quarter of the cycle ahead ( $90^{\circ}$ out of phase). Previous studies have unambiguously demonstrated that learning and stabilization of this movement is facilitated by providing artificially augmented visual feedback (AVF) online during movement execution (Lee et al., 1995; Swinnen et al., 1997; Debaere et al., 2003, 2004): whereas the between-hand phase variability of performers will decrease across practice, the variability of those who receive AVF will decrease faster and to a smaller asymptote. We investigated whether the facilitation provided by AVF results from the integration between visual and proprioceptive afferent information and reflects some optimality principles (MLE) by testing the following predictions: (1) The variability of the pattern with both AVF and proprioception available would reach an optimal level and thus be smaller than with only one modality. (2) If AVF provides less and less salient information about the movement, it would be gradually disregarded. (3) If the AVF is imperceptibly phase shifted, the movement would be partly adapted to compensate for this bias, as the integrated estimate depends partly on the corrupted AVF.

Since the task was learned through practice, we particularly 


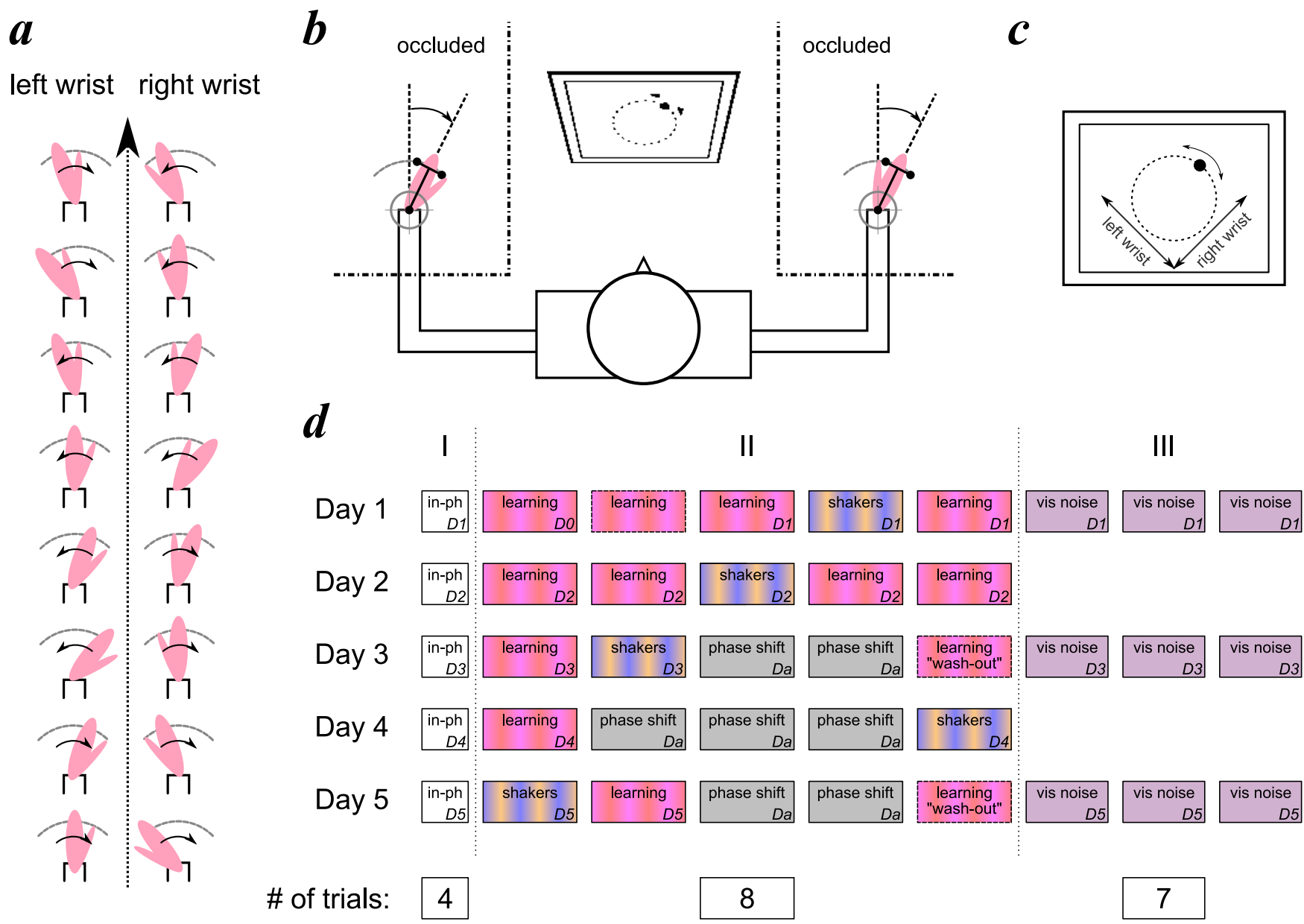

Figure 1. Experimental setup and protocol. $\boldsymbol{a}$, Target movement: the performer had to continuously move both wrists back and forth, being required to maintain $90^{\circ}$ out of phase (a quarter of the cycle) between them. $\boldsymbol{b}$, Sketch of the experimental setup. $\boldsymbol{c}$, During some trials, AVF was provided on a computer screen in front of the performer, by displaying the position of both wrists as the orthogonal coordinates of a single cursor. Perfect $90^{\circ}$-out-of-phase cyclical movement corresponds to a circular trajectory on the screen. $\boldsymbol{d}$, Experimental protocol. The daily experimental session was divided into three parts, delimited by the vertical lines. Part I consisted of a single block (white) of four trials during which subjects had to perform in-phase cyclical movements, without AVF. In parts II and III, the target movement was the $90^{\circ}$ out of phase. Part II consisted of four "learning" (red-magenta) or "phase-shift" (gray) blocks, each containing eight trials during which subject received AVF during four randomly selected trials (50\%). In the "phase-shift" blocks, an artificial phase shift was inserted between the actual movement and the visual feedback, when present. Randomly inserted between these four blocks, part II also contained a "shakers" block (blue- orange) with four trials during which the subject received AVF and four trials without, and had shakers fixed onto the dorsal and palmar section (flexors and extensors) of both wrists. Finally, part III was done on days 1,3 , and 5 and consisted of three "visual noise" blocks, each containing seven trials during which AVF was provided but corrupted by noise, whose level was randomly selected for each trial.

focused on the time course of multisensory integration. Specifically, we investigated whether the model-based predictions were equally validated at the different learning stages: MLE would either be reached after some practice, e.g., with a similar time course to the task, or be acquired much more rapidly. If so, multisensory integration reflecting optimality principles (MLE) would be identified as a process that occurs independently of expertise level in task execution.

\section{Materials and Methods}

Subjects. Fourteen healthy subjects ( 7 females, 7 males) participated (13 were right handed, 1 left handed according to Oldfield's handedness questionnaire). Their age ranged between 18 and 35 (mean 23). All subjects were naive with respect to the experimental goals and were paid for participation. The experimental procedures were approved by the ethical committee of Katholieke Universiteit Leuven, in compliance with the declaration of Helsinki.

Apparatus and task. Subjects were seated in front of a computer screen (Fig. 1b). They inserted both hands into two rotating manipulanda, with the palm in neutral position (thumb upward). Both wrists were free to move with their axis of rotation being aligned with the rotational degree of freedom of both manipulanda. The units were fitted with a forearm rest to support it in a natural position, and the forearms, wrists, and hands were occluded from direct vision.

Performers had to coordinate cyclical oscillatory movements of their wrists such that the right wrist always led the left wrist by a quarter cycle (Fig. 1a). If the trajectories of both wrists followed a sinusoid, maintaining the phase lag would correspond to $\phi=90^{\circ}$ of phase offset between both wrists. This movement is not intrinsic to the motor system (Kelso, 1995), and requires practice before being properly mastered (Zanone and Kelso, 1992; Swinnen et al., 1997; Debaere et al., 2004). Indeed, this pattern is located between the two most natural coordination patterns, which act as strong attractors in the movement state space (Haken et al., 1985; Kelso, 1995; Swinnen, 2002): in-phase corresponds to simultaneous activation of homologous muscles $\left(\phi=0^{\circ}\right)$, and anti-phase corresponds to simultaneous activation of nonhomologous muscles $(\phi=$ $180^{\circ}$ ) or isodirectional movements in extrinsic space when moving in the mediolateral plane. Accordingly, the $90^{\circ}$-out-of-phase pattern studied here was located exactly in between both aforementioned patterns.

During some trials (see below), a black cursor was displayed on the computer screen (Fig. 1b). Subjects were informed that they controlled the moving cursor by their wrists, each of them moving the cursor along 
no compensation

half compensation

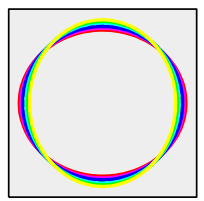

what subject did and felt
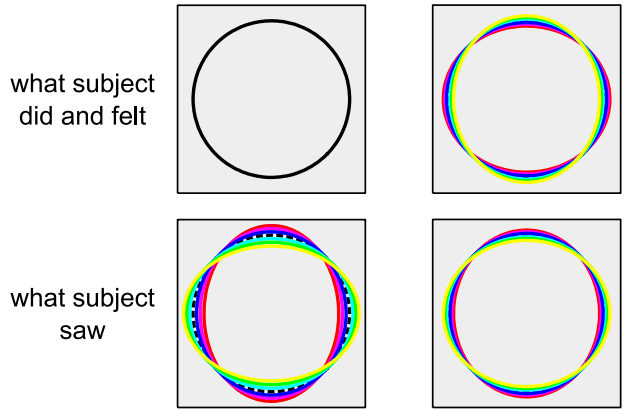

full compensation
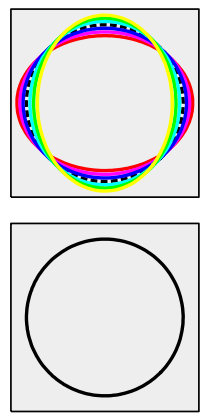

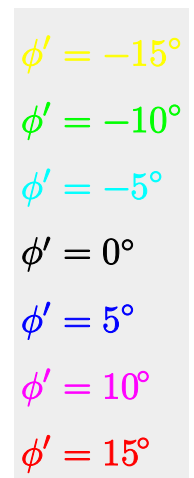

"shakers" blocks also contained four trials (always including the first one) in which the subject received AVF and four trials without AVF, the seven last ones being randomly distributed between trials with and without AVF.

The "phase-shifted" blocks (displayed in gray in Fig. 1d) were inserted to establish the relevance of the third prediction. These blocks were very similar to the learning blocks, except that, when present, the AVF was slightly phase shifted with respect to the actual movement. For example, if the phase shift was $10^{\circ}$, the subject saw an ellipse corresponding to the $100^{\circ}$ out-of-phase pattern on the screen when actually executing the $90^{\circ}$-out-of-phase pattern. Hence, the subject would have to execute the $80^{\circ}$-out-of-phase pattern to see the expected circle on the screen (see supplemental Movie 2, available at www.jneurosci.org as supplemental material). A set of alternative predictions about the subject's behavior in the reference frame of the visual feedback is proposed in Figure 2, depending on the introduced phase shift and the

one coordinate of an orthogonal frame (Fig. 1c), such that the positions of both wrists were integrated in a single visual gestalt (Lissajous figure). Successful $90^{\circ}$-out-of-phase performance was then characterized by tracing an anti-clockwise circle on the screen (see supplemental Movie 1, available at www.jneurosci.org as supplemental material). Three righthanded subjects showed a clear preference for performing the movement with the left wrist ahead (corresponding to a clockwise circle on the screen), and these data were mirrored in the reported analysis. As soon as the first circles appeared on the screen, actual data acquisition started according to the protocol depicted in Figure $1 d$. The whole experiment consisted of five sessions of $\sim 1$ h each, which were completed over 4 or 5 consecutive days. Each session was divided into three parts (see below), each containing some blocks of trials, the duration of each trial being $30 \mathrm{~s}$. Regardless of trial condition, movement frequency was softly constrained around $1 \mathrm{~Hz}$ ( 1 full arm cycle per second) by changing the background color of the screen as soon as the time elapsing between two successive minima of any wrist trajectory deviated from the target period by $>10 \%$.

The experimental session consisted of three parts. Part I consisted of a single block of four trials during which the participant performed in-phase cyclical movements $\left(\phi=0^{\circ}\right)$, without AVF. This control condition was included to assess whether learning the new $90^{\circ}$-outof-phase movement would induce any change in performance from the most stable bimanual coordination pattern, i.e., the mirrorsymmetric in-phase movement.

In parts II and III, the target movement was the $90^{\circ}$-out-of-phase pattern described above $\left(\phi=90^{\circ}\right)$. Part II consisted of four "learning" or "phase-shifted" (see below) blocks, each containing eight trials. During the first and three other (randomly selected) trials (i.e., 50\% of the total), the cursor was visible on the screen, such that AVF was provided. In the remaining four trials, the cursor disappeared after $2 \mathrm{~s}$. Part II also contained a "shakers" block for which we fixated tendon shakers onto the dorsal and palmar region of both subject's wrists, to degrade the quality of the proprioceptive feedback (Bock et al., 2007). While unilateral tendon vibration has been extensively used to bias the proprioceptive inflows toward either flexion or extension (the movement illusion effect) (McCloskey et al., 1983; Casini et al., 2006; Weerakkody et al., 2007), Gilhodes et al. (1986) showed that stimulation of both antagonistic muscles at the same frequency (as we did here) did not induce any overt motor effects. For that reason, simultaneous vibration of agonist and antagonist muscles may provide a useful method to degrade proprioceptive responsiveness, without directly biasing motor output. The shakers' vibration frequency was tuned to $80 \mathrm{~Hz}$, corresponding to the upper limit of Ia afferents firing harmonically with the vibration (Roll et al., 1989). This "shakers" block was randomly inserted in between the four remaining blocks, with the restriction that it was always at least after the third normal block on day 1. Similar to the other blocks in Part II, these level of compensation in the coordination pattern. Importantly, these blocks replaced normal learning blocks without prior warning, such that the phase shift remained undetected by the performer and the corresponding motor adaption occurred unconsciously. Postexperiment interviews revealed that eight subjects did not perceive any discrepancy between their movements and the visual display, while six might have detected the biggest phase shift. Importantly, all the latter subjects thought that the perceived mismatch was due to weak performance and not the result of experimental trickery. The phase shift was implemented as follows: assuming sinusoidal trajectories, let $\theta_{\mathrm{r}}=A \sin (\omega t)$ and $\theta_{1}=A$ $\sin (\omega t+\phi)$ denote the angular trajectories of the right and left wrists, respectively. $A$ and $\omega$ represent the movement amplitude and frequency, and $\phi$ is the phase difference between both wrists. Displaying now the following quantities: $\theta_{\mathrm{r}}^{\text {disp }}=\cos \left(\phi^{\prime} / 2\right) \theta_{\mathrm{r}}-\sin \left(\phi^{\prime} / 2\right) \dot{\theta}_{\mathrm{r}} / \omega$ and $\theta_{1}^{\text {disp }}=$ $\cos \left(\phi^{\prime} / 2\right) \theta_{1}+\sin \left(\phi^{\prime} / 2\right) \dot{\theta}_{1} / \omega-$ calculated on the basis of online estimates of the velocities $\dot{\theta}_{\mathrm{r}}$ and $\dot{\theta}_{1}$-it can be shown that $\theta_{\mathrm{r}}^{\text {disp }}=A \sin \left(\omega t-\phi^{\prime} / 2\right)$ and $\theta_{1}^{\text {disp }}=A \sin \left(\omega t+\phi+\phi^{\prime} / 2\right)$, such that a supplemental phase shift of $\phi^{\prime}$ is artificially introduced in the visual display. We tested visual phase shifts equal to $\phi^{\prime}=-15^{\circ},-10^{\circ},-5^{\circ}, 5^{\circ}, 10^{\circ}$, and $15^{\circ}$. Five trials of each were randomly inserted into the seven "phase-shift" blocks, which still contained $\sim 50 \%$ of trials without visual feedback, to preserve the subjects' belief that these blocks were normal. Those last trials were not recorded for analysis. Note that each phase shift was applied for only $30 \mathrm{~s}$ (the duration of one trial), such that we expect this time to be too small to induce sensory recalibration (see e.g., Burge et al., 2008). On days 3 and 5 , the last learning block was kept normal to wash out any potential effect of the phase-shift blocks on the degree of certainty of the AVF, before starting part III of the experimental session. These "wash-out" blocks were not included in the analyses.

Part III was done on days 1, 3, and 5 and consisted of three "visual noise" blocks, each containing seven trials in which AVF was provided but corrupted by noise. In those blocks, the displayed signals were $\theta_{\mathrm{r}}^{\text {disp }}=$ $\alpha \theta_{\mathrm{r}}+\lambda_{\mathrm{r}}$ and $\theta_{1}^{\text {disp }}=\alpha \theta_{1}+\lambda_{\mathrm{l}}, \alpha$ weighting the presence of the actual movement in the display, and $\lambda_{\mathrm{r}}$ and $\lambda_{1}$ being two independent noise vectors obeying the dynamics of a damped spring excited by a random force of Gaussian distribution (see supplemental Movie 3, available at www.jneurosci.org as supplemental material). In discrete time, their dynamics then obeyed $\lambda[k]=\left(-\lambda[k-2]+(2+\xi \tau) \lambda[k-1]+\tau^{2} \mu[k]\right) /$ $\left(1+\xi \tau+\kappa \tau^{2}\right)$, where $\xi=10, \kappa=100$, and $\tau=0.005$ s are the damping and stiffness of the spring, and the display refresh rate, respectively. The force acting on this virtual spring-i.e., $\mu[k]$ — was a random vector of Gaussian distribution (zero mean, $\Lambda$ variance). Six of the seven trials per block were tuned as follows: $\alpha=1$ and $\Lambda=2500,5000,7500,10,000$, 12,500 , and 15,000 , such that different noise levels were superimposed on the actual signal. The seventh trial was tuned with $\alpha=0$ and $\Lambda=30,000$, 
such that the visual display was uncorrelated with the actual movement. The seven conditions were randomized within each block.

A "day" label is indicated on the bottom-right corner of many blocks in Figure 1d: $D 0$ refers to the block whose data represented the initial level, $D 1-D 5$ refer to the blocks whose data were preserved to represent the skill levels from days 1 to 5; and $D a$ stands for the phase-shifted blocks, whose data were merged together for the corresponding analysis. The blocks without $D$ label were not preserved for analysis.

Data analysis. The first $3 \mathrm{~s}$ of data from each trial were removed since we were not interested in movement initiation. The angular position of both wrists was filtered by a Butterworth filter (forward and backward, cutoff frequency of $8 \mathrm{~Hz}$ ). They were also detrended by subtracting the best-fitting second-order parabola, to remove any low-frequency drift. Angular velocity of the wrists was computed by an appropriate centered differentiation algorithm.

The movement frequency was calculated as the mean of the inverse of the time elapsed between two adjacent maxima in the trajectory, then averaged over both wrists. The continuous phase difference between both wrists was calculated as follows:

$$
\phi=\arctan \left(\frac{\theta_{\mathrm{r}} \dot{\theta}_{\mathrm{l}}-\theta_{1} \dot{\theta}_{\mathrm{r}}}{\frac{\dot{\theta}_{\mathrm{r}} \dot{\theta}_{\mathrm{l}}}{2 \pi f}+2 \pi f \theta_{\mathrm{r}} \theta_{\mathrm{l}}}\right),
$$

where $\theta_{\mathrm{r}}, \dot{\theta}_{\mathrm{r}}, \theta_{1}$, and $\dot{\theta}_{1}$ denote the position and velocity of the right and left wrists, and $\bar{f}$ is the mean movement frequency over the corresponding trial. The mean and SD of $\phi$ over a trial were calculated according to circular statistics standards (Fisher, 1983):

$$
\begin{gathered}
\mu_{\phi}=\arg \left(\int_{0}^{T} e^{i \phi(t)} d t\right), \\
\sigma_{\phi}=\sqrt{-2 \ln \left\|\frac{\int_{0}^{T} e^{i \phi(t)} d t \|}{T}\right\|},
\end{gathered}
$$

where $T$ is the trial duration. The mean of the estimate of the phase difference between both wrists-i.e., $\mu_{\phi}$ - whatever the feedback condition, was computed as the mean of the actual phase difference during the corresponding trial. The variance $\sigma^{2}$ was computed as the square of the $\mathrm{SD}$ of this phase difference.

To quantify the learning rate of the task, exponential curves were fitted to the SD of $\phi$ across days of practice. These curves took the following form:

$$
\sigma_{\phi, d}=\sigma_{\phi, 1} e^{-\frac{d-1}{\delta}}+\sigma_{\phi, \infty},
$$

where $d$ stands for the day considered. The three parameters of Equation 4 -i.e., the asymptote $\sigma_{\phi, \infty}$, the decrease amplitude $\sigma_{\phi, 1}$, and the decrease time constants (in days) $\delta$-were estimated by a nonlinear leastsquares curve-fitting algorithm (The MathWorks).

Influence of practice and type of feedback provided was assessed by factorial ANOVA designs. The level of significance was set to $p<0.05$.

Maximum likelihood model. A model assuming the integration between proprioception and AVF computes the integrated estimate of the phase difference between both limbs from the estimates given by each modality alone, i.e.:

$$
\mu_{\mathrm{V}+\mathrm{P}}=w_{\mathrm{P}} \mu_{\mathrm{P}}+\left(1-w_{\mathrm{P}}\right) \mu_{\mathrm{V}}
$$

where $\mu_{\mathrm{P}}$ and $\mu_{\mathrm{V}}$ are the estimates provided by proprioception and visual feedback alone, respectively, and $0 \leq w_{\mathrm{P}} \leq 1$ weights the contribution of the proprioceptive feedback in the global estimate. This model assumes that the integrated variable is linear, while the phase actually lies on a circle. Conversely, the tangent of the phase would be a linear variable, but it can be shown that $\theta \simeq \tan \theta$ for $\theta \in\left[-40^{\circ}, 40^{\circ}\right]$. We assume that the linear model is thus valid for phase deviations around steady state belonging to that range. Given Equation 5, the variance of the integrated estimate is thus $\sigma_{\mathrm{V}+\mathrm{P}}^{2}=w_{\mathrm{P}}^{2} \sigma_{\mathrm{P}}^{2}+\left(1-w_{\mathrm{P}}\right)^{2} \sigma_{\mathrm{V}}^{2}$, assuming that $\sigma_{\mathrm{P}}^{2}$ and $\sigma_{\mathrm{V}}^{2}$ denote the variance of the proprioceptive and visual feedback, respectively, and that both are corrupted by independent noise exhibiting Gaussian distribution. The model is optimal if it maximizes the likelihood of the integrated signal by selecting $w_{\mathrm{P}}$ to minimize the variance $\sigma_{\mathrm{V}+\mathrm{P}}^{2}$, such that the average square error-i.e., $\left(\mu_{\mathrm{V}+\mathrm{P}}-90^{\circ}\right)^{2}$-is minimized through the trial. It can be demonstrated (see the references above) that $\sigma_{\mathrm{V}+\mathrm{P}}^{2}$ is minimized if the following is true:

$$
w_{\mathrm{P}}=\frac{\sigma_{\mathrm{V}}^{2}}{\sigma_{\mathrm{P}}^{2}+\sigma_{\mathrm{V}}^{2}},
$$

i.e., if the weight of each modality is inversely proportional to its own variance. Accordingly, the variability of the optimally integrated estimate is equal to the following:

$$
\sigma_{\mathrm{V}+\mathrm{P}}^{2}=\frac{\sigma_{\mathrm{V}}^{2} \sigma_{\mathrm{P}}^{2}}{\sigma_{\mathrm{V}}^{2}+\sigma_{\mathrm{P}}^{2}},
$$

and is smaller than the variability of either modality alone $\left[\sigma_{\mathrm{V}+\mathrm{P}}^{2}<\right.$ $\left.\min \left(\sigma_{\mathrm{P}}^{2}, \sigma_{\mathrm{V}}^{2}\right)\right]$, except if one sensory source is perfect $\left(\sigma^{2}=0\right)$ or is infinitely noisy $\left(\sigma^{2}=\infty\right)$. The maximal advantage of multisensory combination is obtained when both modalities are of equal variance, since the variance of their integration becomes $\sqrt{2}$ times smaller (78\%).

This simple model cannot be directly applied to our data as such, for the following two reasons: (1) the variability corresponding to the AVFi.e., $\sigma_{\mathrm{V}}^{2}$-cannot be estimated, since it would correspond to a condition in which all proprioceptive afferents are shut down, while the tendon shakers used in this study only increased the noise level on some of the proprioceptive afferents (Bock et al., 2007); (2) the $90^{\circ}$-out-of-phase pattern is most likely stabilized by a mix between feedback-driven motor commands - based on the proprioceptive and visual inflows- and feedforward motor commands, which are generated from internal predictions about the system's dynamical evolution (Wolpert et al., 1998; Kawato, 1999; Sabes, 2000), and whose influence has been neglected in the model (Eq. 7). For these reasons, we augmented the two-component model (Eq. 7) to a three-component model, integrating the sensory inflows coming from the visual system $(\mathrm{V})$, the proprioceptive system, which is impaired by the shakers ( $\mathrm{P}$ for short), and the sum of the residual proprioceptive information and the feedforward command (FF for short). Similarly to what has been done for the two-component model, it can be shown that optimal integration of those three components into a common belief about the phase offset would weight each modality according to the following:

$$
\begin{gathered}
w_{\mathrm{V}}=\frac{\sigma_{\mathrm{P}}^{2} \sigma_{\mathrm{FF}}^{2}}{\sigma_{\mathrm{V}}^{2} \sigma_{\mathrm{P}}^{2}+\sigma_{\mathrm{V}}^{2} \sigma_{\mathrm{FF}}^{2}+\sigma_{\mathrm{P}}^{2} \sigma_{\mathrm{FF}}^{2}}, \\
w_{\mathrm{P}}=\frac{\sigma_{\mathrm{V}}^{2} \sigma_{\mathrm{FF}}^{2}}{\sigma_{\mathrm{V}}^{2} \sigma_{\mathrm{P}}^{2}+\sigma_{\mathrm{V}}^{2} \sigma_{\mathrm{FF}}^{2}+\sigma_{\mathrm{P}}^{2} \sigma_{\mathrm{FF}}^{2}}, \\
w_{\mathrm{FF}}=\frac{\sigma_{\mathrm{V}}^{2} \sigma_{\mathrm{P}}^{2}}{\sigma_{\mathrm{V}}^{2} \sigma_{\mathrm{P}}^{2}+\sigma_{\mathrm{V}}^{2} \sigma_{\mathrm{FF}}^{2}+\sigma_{\mathrm{P}}^{2} \sigma_{\mathrm{FF}}^{2}},
\end{gathered}
$$

and that the resulting variability would be equal to the following:

$$
\sigma_{\mathrm{V}+\mathrm{P}+\mathrm{FF}}^{2}=\frac{\sigma_{\mathrm{V}}^{2} \sigma_{\mathrm{P}}^{2} \sigma_{\mathrm{FF}}^{2}}{\sigma_{\mathrm{V}}^{2} \sigma_{\mathrm{P}}^{2}+\sigma_{\mathrm{V}}^{2} \sigma_{\mathrm{FF}}^{2}+\sigma_{\mathrm{P}}^{2} \sigma_{\mathrm{FF}}^{2}} .
$$

Therefore, the three conditions corresponding to the trials without $\mathrm{AVF}$ and/or with the tendon shakers can be used to compute the sensory variability of each source alone, i.e., $\sigma_{\mathrm{V}}^{2}, \sigma_{\mathrm{P}}^{2}$, and $\sigma_{\mathrm{FF}}^{2}$, assuming the following restrictions: (1) the performance variability (i.e., the variability measured from Eq. 3) fairly reflects the sensory estimation variability; and (2) the maximum likelihood estimator is appropriate to model the stationary performance, while an optimal control model—which would need to consider e.g., the task's dynamics-might be a more complete model of the behavior (see Discussion). If the available information sources are optimally integrated, we find the following: $\hat{\sigma}_{\mathrm{BS}}^{2}=\sigma_{\mathrm{FF}}^{2}, \hat{\sigma}_{\mathrm{B}}^{2}=$ $\sigma_{\mathrm{P}}^{2} \sigma_{\mathrm{FF}}^{2} /\left(\sigma_{\mathrm{P}}^{2}+\sigma_{\mathrm{FF}}^{2}\right)$, and $\hat{\sigma}_{\mathrm{S}}^{2}=\sigma_{\mathrm{V}}^{2} \sigma_{\mathrm{FF}}^{2} /\left(\sigma_{\mathrm{V}}^{2}+\sigma_{\mathrm{FF}}^{2}\right)$, where $\hat{\sigma}_{\mathrm{BS}}^{2}, \hat{\sigma}_{\mathrm{B}}^{2}$, and $\hat{\sigma}_{\mathrm{S}}^{2}$ 

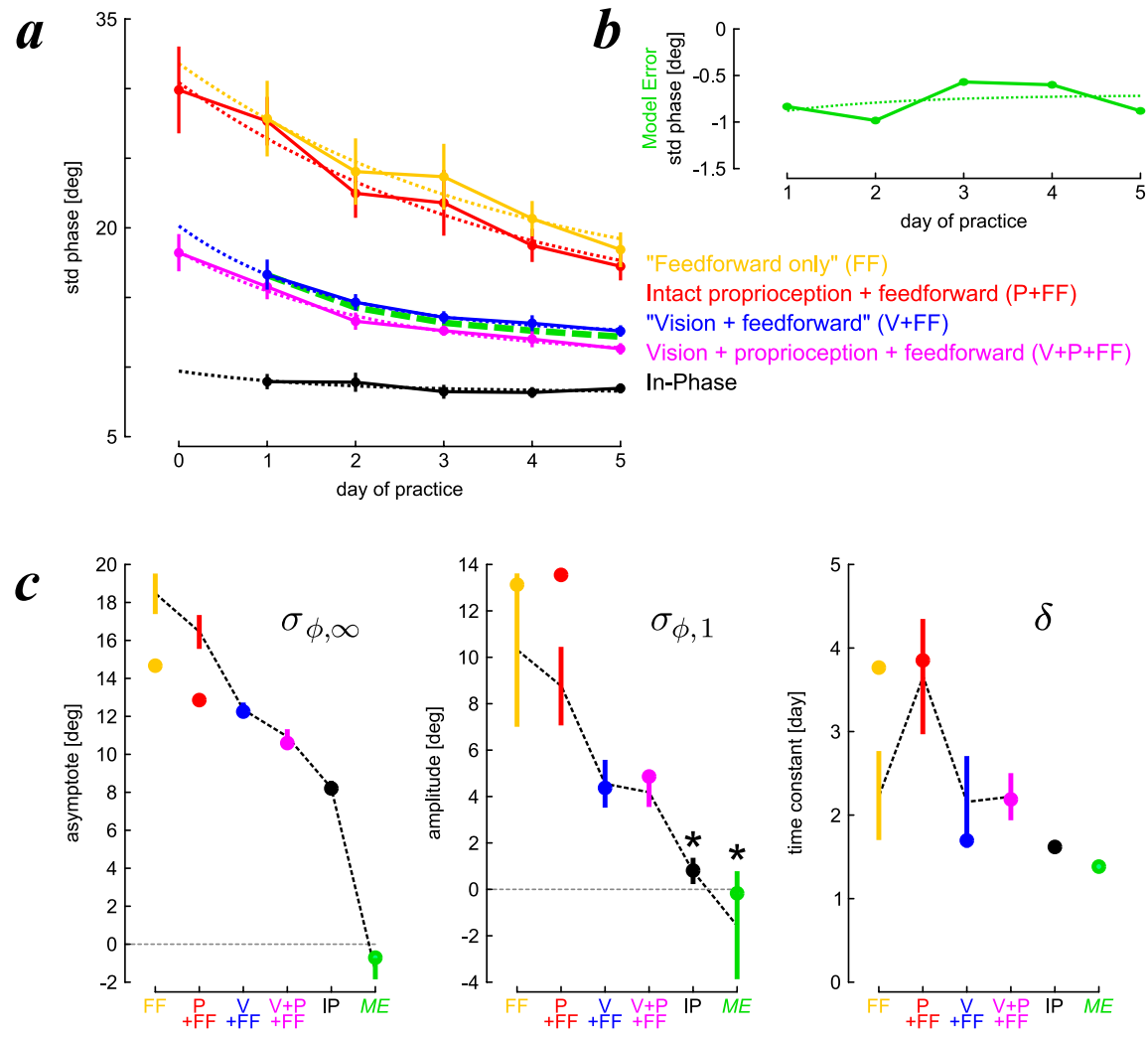

Figure 3. Evolution of task stability across practice and types of task and feedback. The error bars represent the betweensubjects SE. $\boldsymbol{a}$, SD (std) of the phase difference between both wrists across the $5 \mathrm{~d}$ of practice for the in-phase movement (black), the "feedforward only" (orange), the intact proprioception + feedforward (red), the "vision + feedforward" (blue), and the proprioception + vision + feedforward (magenta) conditions. The dotted lines are best exponential fits (Eq. 4). The model-based prediction on the optimal level to reach with the three feedback modalities is displayed in green (dashed). It has been calculated from estimates of the single-modality levels from the actual measurements in the three other conditions, according to Equation 10. $\boldsymbol{b}$, Model error (ME): difference between actual task variability with the three feedback modalities (the magenta curve in $\boldsymbol{a}$ ) and the model-based prediction (the dashed green curve in $\boldsymbol{a}$ ). The dotted line is the best exponential fit (Eq. 4). $\boldsymbol{c}$, Parameters of the exponential fits: asymptote $\left(\sigma_{\phi, \infty}\right)$, amplitude $\left(\sigma_{\phi, 1}\right)$, and time constant $(\delta)$ for the 5 conditions displayed in $\boldsymbol{a}$, and the ME displayed in $\boldsymbol{b}$. The dots represent the group average, and the error bar the mean $\pm \mathrm{SE}$ for individual fits. The asterisks highlight the amplitude fits that are not significantly different from zero ( $t$ tests, $p>0.18$ ).

refer to the performance variability that was measured in the trials without AVF and with the shakers (blind+shakers), without AVF and shakers (blind only), and with AVF and shakers (shakers only), respectively. The equations can be inverted to obtain an estimate for $\sigma_{\mathrm{V}}^{2}, \sigma_{\mathrm{P}}^{2}$, and $\sigma_{\mathrm{FF}}^{2}$. From there, a model-based prediction about the performance variability with both intact proprioception and AVF can be estimated from Equation 9 and compared with the actual data:

$$
\sigma_{\mathrm{V}+\mathrm{P}+\mathrm{FF}}^{2}=\frac{\hat{\sigma}_{\mathrm{S}}^{2} \hat{\sigma}_{\mathrm{B}}^{2} \hat{\sigma}_{\mathrm{BS}}^{2}}{\hat{\sigma}_{\mathrm{S}}^{2} \hat{\sigma}_{\mathrm{BS}}^{2}+\hat{\sigma}_{\mathrm{B}}^{2} \hat{\sigma}_{\mathrm{BS}}^{2}-\hat{\sigma}_{\mathrm{S}}^{2} \hat{\sigma}_{\mathrm{B}}^{2}} .
$$

Specifically, the model-based predictions can now be rephrased as follows, on the basis of the derived equations.

(1) The variability of performance with both AVF and intact proprioception available is smaller than with any other sensory combination, and variability should reach the optimal level predicted by the maximum likelihood integrator (Eq. 9 or 10).

(2) If the AVF is artificially corrupted by noise (such that $\sigma_{\mathrm{V}}^{2}$ increases), the movement variability increases but saturates toward the level without AVF. Indeed, according to Equation 9, if $\sigma_{\mathrm{V}}^{2}$ goes to infinity, $\sigma_{\mathrm{V}+\mathrm{P}+\mathrm{FF}}^{2}$ tends to-and never exceeds- $\sigma_{\mathrm{P}+\mathrm{FF}}^{2}=\sigma_{\mathrm{P}}^{2} \sigma_{\mathrm{FF}}^{2} /\left(\sigma_{\mathrm{P}}^{2}+\sigma_{\mathrm{FF}}^{2}\right)$.

(3) If the AVF is imperceptibly phase shifted with respect to the actual movement (such that $\mu_{\mathrm{V}}$ does not equal $\mu_{\mathrm{P}}$ anymore), the movement is partly adapted to compensate for this bias, since the integrated estimate depends on both the uncorrupted (feedforward+proprioception) and the corrupted (AVF) modalities (see Eq. 5). The extent of this compen- sation could be derived from the weights assigned to the different modalities by the maximum likelihood integrator model (Eq. 8).

\section{Results}

The following sections will sequentially address the results related to the three model-based predictions.

\section{Prediction 1}

The first prediction was validated by comparison of the four different feedback conditions: intact proprioception + feedforward (visual display not provided), proprioception+ vision + feedforward (visual display provided), "vision + feedforward" (with distorted proprioception), and "feedforward only" (visual display not provided and distorted proprioception). Since proprioception cannot be reversibly eliminated without invasive procedures, proprioceptive inflows in the two latter conditions were distorted by placing tendon shakers on the palmar and dorsal side of both wrists (Bock et al., 2007). The results are displayed in Figure 3. Figure $3 a$ displays the SD of the phase difference between both wrists across the $5 \mathrm{~d}$ of practice and the five types of movements [in-phase, "feedforward only" (with the shakers and no AVF), intact proprioception and feedforward (no AVF), "vision and feedforward" (with the shakers and AVF), and with the three modalities (no shakers, with AVF)].It shows a clear tendency to reduce the SD_-or increase stability — with practice under the four movement conditions corresponding to the $90^{\circ}$-out-of-phase pattern, while in-phase pattern stability was very constant across days. Factorial ANOVA $(5 \times 5$, five days, five movement types: four conditions with the $90^{\circ}$-out-of-phase pattern, and in-phase) supported this finding as shown by a significant practice day $\operatorname{effect}\left(F_{(4,325)}=14.9, p<0.0001\right)$. The movement type effect also reached significance $\left(F_{(4,325)}=11.24, p<0.0001\right)$, suggesting that the task was most difficult to stabilize without AVF and with the shakers (i.e., "feedforward only," orange), then without AVF (proprioception+feedforward, red), then with the shakers ("vision+feedforward," blue), and finally with the three modalities available and uncorrupted (magenta). The in-phase pattern was more successfully stabilized than any of the latter four conditions. One-way ANOVA on the data corresponding only to in-phase movements (with the day of practice as single factor) did not reach significance $(p>0.7)$, suggesting that the extensive practice of the $90^{\circ}$-out-of-phase pattern under various feedback conditions did not modify the performance level of the control in-phase condition.

The qualitative aspect of prediction 1 is clearly visible in Figure $3 a$ : the more feedback the subjects received, the more stable their performance. This result suggests that the integration between the available modalities (proprioceptive feedback, AVF, and internally generated feedforward command) reflects some maximum likelihood mechanisms. However, the shakers' effect was small: while it reached significance with AVF [the difference between the blue and the magenta curves in Fig. $3 a$ was assessed by 


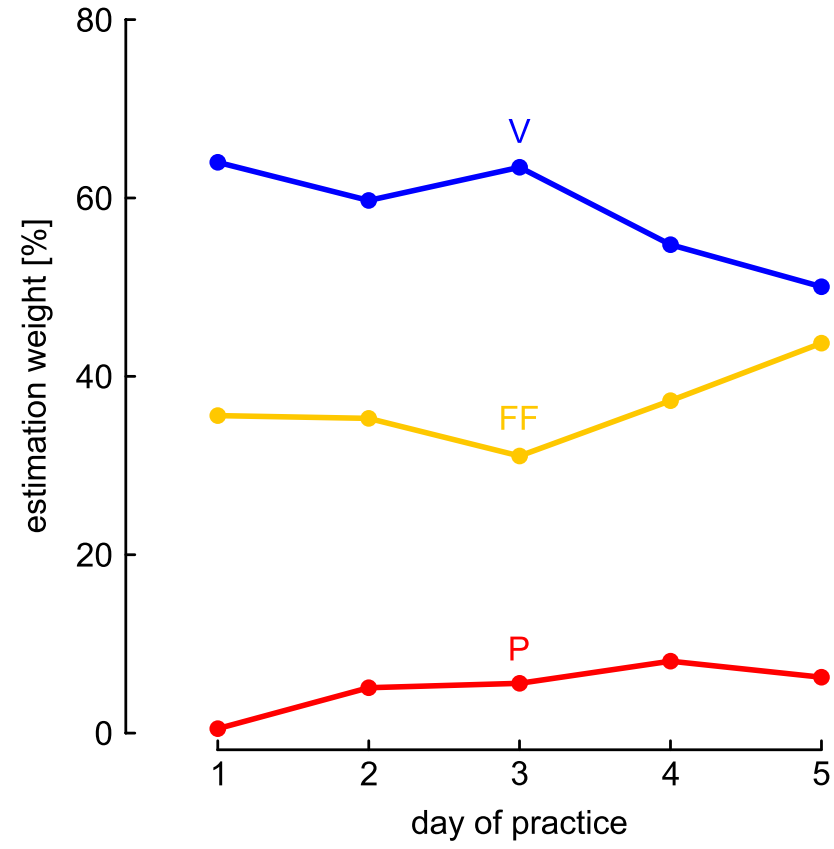

Figure 4. Evolution of the weights assigned to each modality [proprioception (P), augmented visual feedback (V), and feedforward (FF)] across the $5 \mathrm{~d}$ of practice. These weights are inferred from the actual measurements, according to Equation 8.

a dedicated factorial ANOVA that reached significance with $\left.F_{(1,130)}=7.8(p<0.01)\right]$, it did not reach significance between the two conditions where AVF was not provided [the difference between the orange and the red curves in Fig. $3 a$ was assessed by a dedicated factorial ANOVA that did not reach significance $(p>$ $0.2)$ ]. This result suggests that quantitative matching of maximum likelihood predictions would require other techniques to entirely shut down the proprioceptive afferents. Those techniques are, however, more invasive.

Performance variability could actually be predicted from the three other measurements (see Eq. 10). This prediction is depicted with the dashed green curve in Figure $3 a$, while Figure $3 b$ represents the difference between this prediction and the actual performance variability (the magenta curve in Fig. $3 a$ ). Visual inspection of the figure reveals that the model-based prediction underestimated the actual performance by $\sim 1^{\circ}$ (or $\sim 8 \%$ of the asymptotic variability). Indeed, since the data suggested that the shakers' effect was tiny without AVF, the model predicts an even smaller effect when combined with another sensory modality, i.e., AVF. The weight assigned to the different modalities could be estimated from Equation 8. These weights are displayed in Figure 4, which suggests that the weight assigned to the AVF slightly decreased in the final learning stages (days 3-5) in favor of the weights assigned to the proprioceptive and internal (feedforward) modalities.

To further investigate the differences in the learning time courses, we fitted exponential learning curves onto the data of Figure 3, $a$ and $b$ (dotted lines). The equation of these fits is given by Equation 4 and contains three parameters that were optimized for the group data and for each subject individually. In Figure $3 c$, the large dots denote the group data, and the error bars denote the mean \pm SE of the individual fits. The three fitted parameters are as follows: (1) the asymptote $\sigma_{\phi, \infty}$, i.e., the level reached at the end of the learning; (2) the amplitude $\sigma_{\phi, 1}$, i.e., the difference between the level at the first day of practice and the asymptote; (3) and the time constant $\delta$, characterizing the learning speed. Given the as- ymptotes, the figure confirms that the $90^{\circ}$-out-phase pattern was more difficult to stabilize with the tendon shakers on the wrists than without, and even more difficult when the visual feedback was not provided. The in-phase movement was the most stable one. The asymptote of the difference between the proprioception + vision condition and the model-based prediction (green) reached a value about -0.7 , confirming that the actual data outperformed the model-based prediction by this small amount. More interestingly, this figure shows that two amplitude parameters were not significantly different from zero ( $t$ tests, $p>0.18$ ): (1) the amplitude of the model-error fit (green) was not different from zero, revealing that this fit was equally close to zero over the whole learning course; (2) the amplitude of fit made on the in-phase data (black) was also zero, confirming that performance in this condition was stable, its variability being constant across the $5 \mathrm{~d}$. The time constants of the conditions that were actually learned through practice (i.e., the four movement conditions stabilizing the $90^{\circ}$-out-of-phase pattern that corresponded to nonzero amplitude) were $\sim 2-4 \mathrm{~d}$. The time constants of the individual fits for the in-phase movement and the difference between the model prediction and the "all modalities" condition were not displayed, since the learning amplitude was not different from zero in both cases. In sum, the performers learned to stabilize the $90^{\circ}$-out-of-phase movement along the $5 \mathrm{~d}$ of practice, regardless of the available sensory modality(ies), but it seems that their integration was equally close to the predicted optimal level from the first to the last session.

\section{Prediction 2}

The second prediction was investigated through specific blocks, executed at the end of sessions 1,3, and 5. Here, the AVF was always present, but corrupted by some degree of noise: on top of the actual movement, the cursor obeyed the dynamics of a randomly actuated damped spring (see Materials and Methods). The noise strength varied across six levels, and a seventh condition was added in which the cursor moved completely randomly, i.e., uncorrelated with the actual movement. The SDs of the phase difference between both wrists in these noisy conditions are displayed in Figure 5, together with the "vision+feedforward," the "proprioception+feedforward," and the "vision+ proprioception+feedforward" conditions of the same days (same as in Fig. 3a). As expected, increasing the noise level resulted in a performance deterioration: factorial ANOVA $(3 \times 8$, three days, eight noise levels including baseline $\mathrm{V}+\mathrm{P}+\mathrm{FF}$ ) reached significance with $F_{(7,312)}=21.1(p<0.001)$ with the noise level as factor. The effect of day of practice also reached significance, due to the learning effect identified previously $\left(F_{(2,312)}=53, p<0.001\right)$, and their interaction with $F_{(14,312)}=2.7(p<0.001)$. More importantly, the variability achieved under all of these noise conditions never exceeded the level reached without any visual feedback (i.e., the P+FF condition). Specifically, the level reached in the "infinitely noisy" condition (no correlation between movement and visual display) was not significantly different from the one reached without visual feedback ( $t$ tests, all $p>0.16)$. This is highly consistent with the second prediction: when the AVF does not provide any salient information about the movement, it is simply disregarded such that performance equals the level obtained without any AVF. The same figure displays qualitative model-based predictions (dotted line): the variability increases smoothly, saturating at the "proprioception + feedforward" $(\mathrm{P}+\mathrm{FF})$ level. Once again, the matching between the prediction and the actual data is of similar quality across days. 


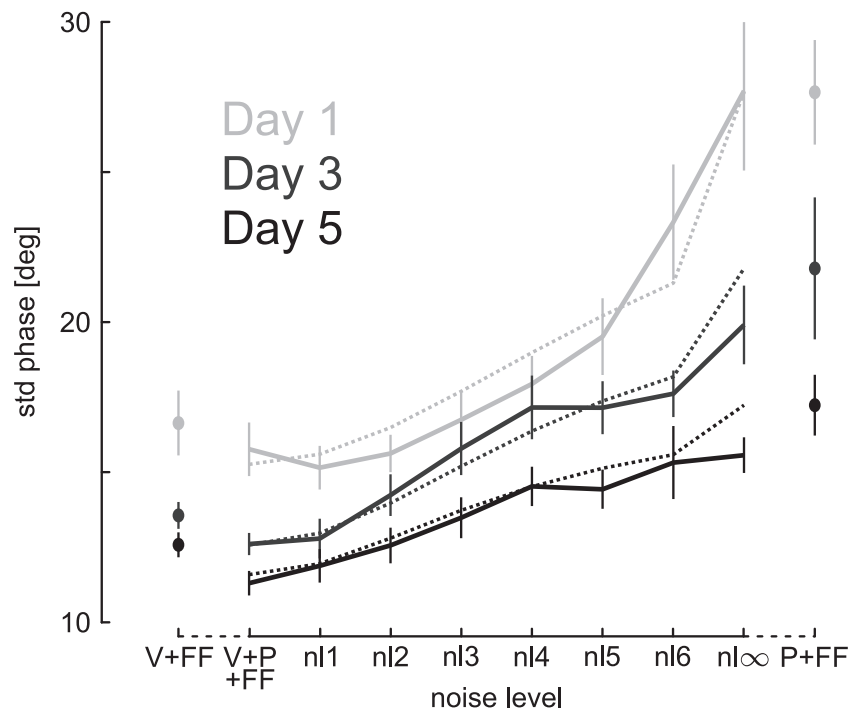

Figure 5. SD (std) of the phase difference between both wrists for the "visual noise" blocks. The "vision + feedforward" (V+FF), vision + proprioception + feedforward (V+P+FF), and proprioception + feedforward $(\mathrm{P}+\mathrm{FF})$ levels reached on the corresponding days are displayed. Between the $V+P+F F$ and $P+F F$ conditions, the SDs reached with the seven noise levels ( $\mathrm{nl}$ ) are displayed. The error bars represent the between-subjects SE. The dotted curves represent model-based predictions, the performance reaching the same level as without AVF when the noise is augmented.

\section{Prediction 3}

To validate the third model-based prediction, we replaced some "normal" learning blocks of days 3,4 , and 5 by specific blocks (see Fig. $1 d$ ) where the visual display (when present) was phase shifted (see Materials and Methods). Importantly, the subjects were not informed about this experimental manipulation, and the introduced phase shifts were kept small enough $\left( \pm 15^{\circ}\right)$ such that they were not perceived. Accordingly, performers did not detect the mismatch between what they did (and felt via proprioceptive feedback) and what they saw, suggesting that any potential movement compensation was thus unconscious. Postexperiment interviews indicated that some subjects did perceive the largest phase shifts, but always attributed the mismatch to inaccurate movement execution. Several possibilities about movement and visual display were presented in Figure 2, depending on the degree of compensation in the coordination pattern. The two extremes were either no compensation (the stabilized movement is always the same, and the AVF is fully skewed), and full compensation (the stabilized movement is fully adapted to correspond to a circular AVF, whatever the phase shift). In Figure $6 a$, the actual average trajectories are displayed, together with the corresponding visual display (AVF). One can see that the subjects partly compensated for the introduced phase shifts, i.e., between the two extremes described above, such that the visual display was also only partly skewed. Correspondingly, Figure $6 b$ represents the phase difference error, i.e., the mean of the phase difference between the two wrists (computed by Eq. 5) normalized by the mean of this difference across conditions. If the phase shifts were not compensated, this line would have been horizontal (the stabilized pattern would have been the same whatever the shape of the visual display). In contrast, full compensation would have led to a diagonal line of slope -1 . The actual data lie in between, suggesting that the weight assigned to AVF was 50\% to build the estimate of the phase difference between both wrists. Partial compensation was validated by an ANOVA, which reached significance with $F_{(6,91)}=57.4(p<0.0001)$, with the phase-shift level as single factor (seven phase shifts were tested). Full compensation was furthermore invalidated by individual $t$ tests showing significant differences between the six data points and the diagonal line of slope -1 (all $p<0.004)$.

The compensatory behavior could have been predicted from the results related to the first prediction, since the three modalities should be combined according to weights inversely proportional to their own variance (see Eq. 8 and Fig. 4). Using the data from the learning blocks on days 3, 4, and 5-i.e., the same days as those where the "phase-shift" blocks were inserted-Figure 4 suggests that the visual feedback should be weighted 50-60\% in the integrated estimate. The predicted compensation is actually represented by the dashed gray line in Figure $6 b$. The figure reveals that the predicted and actual degree of compensation are very close to each other and are not statistically different for the majority of phase shifts (the asterisks, $t$ tests, $p>0.05$ ).

Finally, we investigated whether the introduced phase shifts in the AVF increased task difficulty for the performer. This is unlikely since the pattern variability stayed constant across the tested phase shifts (see Fig. $6 c$ ). ANOVA did not reach significance for this variable $\left(F_{(6,91)}=0.24, p>0.96\right)$, with the phaseshift level as single factor.

\section{Discussion}

Multiple sensory modalities are integrated by the human brain to obtain a common belief about the world state, and multisensorytraining protocols could better approximate natural settings and boost perceptual learning (Shams and Seitz, 2008). Optimal computational models predict that the integrated signal variability will be minimized, if the signal on each modality is weighted with respect to the inverse of its variance, such that the average error between the perceived and the actual world state will be minimized. This optimal integration process is often referred to as maximum likelihood estimation (MLE, assuming all Gaussian likelihoods and symmetrical cost functions). Human capabilities relying on MLE have been broadly demonstrated in the past (Trommershäuser et al., 2008), during decision-making tasks (e.g., source localization) (van Beers et al., 1999; Ernst and Banks, 2002; Alais and Burr, 2004; Helbig and Ernst, 2007; Körding et al., 2007), point-to-point reaching, or saccadic movements (Körding and Wolpert, 2004; Tassinari et al., 2006; Vaziri et al., 2006).

Here, we investigated multisensory integration during the execution of a cyclical bimanual task requiring extensive learning. In particular, the present contribution was twofold. First, we showed that multisensory integration between proprioception and AVF can be performed online, during the execution of a rhythmic movement, while previous studies focused on discrete tasks where multisensory integration and motor action could be separated in time. For example, Sober and Sabes (2003, 2005) illustrated flexible strategies for the integration of proprioceptive and visual feedback, yet restricted to the early stages of movement planning. Second we demonstrated that the integration process was already in place during the initial stage of practice, whereas the task necessitated substantial training before reaching an asymptotic level of performance.

We investigated the performers' capability to reflect integration mechanisms through MLE by testing three predictions that required independent manipulations: (1) integration of the different modalities through learning; (2) corrupting AVF by noise, such that it progressively became less useful; and (3) imperceptibly skewing AVF, such that it provided biased information. Our results confirmed that the $90^{\circ}$-out-of-phase coordination pattern does not belong to the intrinsic bimanual repertoire (Zanone and 
Kelso, 1992; Lee et al., 1995; Swinnen et al., 1997; Debaere et al., 2003, 2004), since its SD was substantially higher than for the in-phase condition, and was only gradually reduced over five daily practice sessions. This was the case regardless of available modalities. Importantly, the phase difference between both limbs is a rather artificial quantity that was most likely not spontaneously encoded by the subjects' brain, although we show that it nicely captures the subjects' performance and reflects multisensory integration mechanisms.

The performance variability that was observed with all three modalities available was slightly better than predicted by the model (by $1^{\circ}$, or $8 \%$ of the asymptotic value), obtained by inferring the variability of each modality alone from the other experimental conditions. This highlights an important concern about the experimental manipulations we introduced to validate the first prediction: the tendon shakers used to reversibly degrade proprioception actually only partly affected the proprioceptive inflow from the corresponding muscle (Roll et al., 1989), without completely masking sensory information. Similarly, cutaneous receptors were unaffected by the shakers, and so could provide uncorrupted timing feedback on turning points (maximum flexion or extension). For those reasons, the "shakers" conditions most likely retained some residual proprioception, and the movement variability in these conditions might have resulted from the exploitation of this remaining inflow. The MLE model was thus less thoroughly tested with tendon shakers than it would have been if proprioception was entirely masked through some more invasive techniques. However, the predicted qualitative trend was observed: the available modalities were exploited to reduce the performance variability. Importantly, the two other predictions, which further strengthened the MLE hypothesis, were not based on tendon vibration conditions.

The experimental manipulations associated with prediction 2 directly impaired AVF quality by adding some noise to its dynamics. The prediction from MLE was that performance should never be worse than without AVF. It might be counterintuitive, since noisy AVF could act as a distractor and induce large errors in the stabilized pattern. This was not the case, and the subjects nicely integrated their proprioceptive inputs with the noisy AVF to optimize their movement stability. In particular, performance with AVF that was uncorrelated with the actual movement (and thus was virtually useless) was not distinguishable from performance with proprioception only. In a recent paper, Burge et al. (2008) found that adding random noise in the visual feedback during reaching movements does not affect the adaptation rate to visual offsets, contrasting with optimal predictions from a Kalman filter. Our results are not incompatible, since here we studied the effect of random noise on steady-state performance vari- $\boldsymbol{b}$

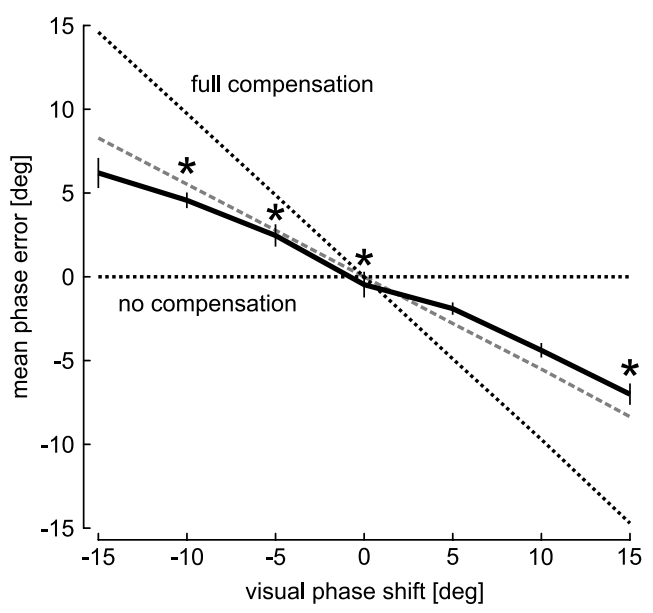

c

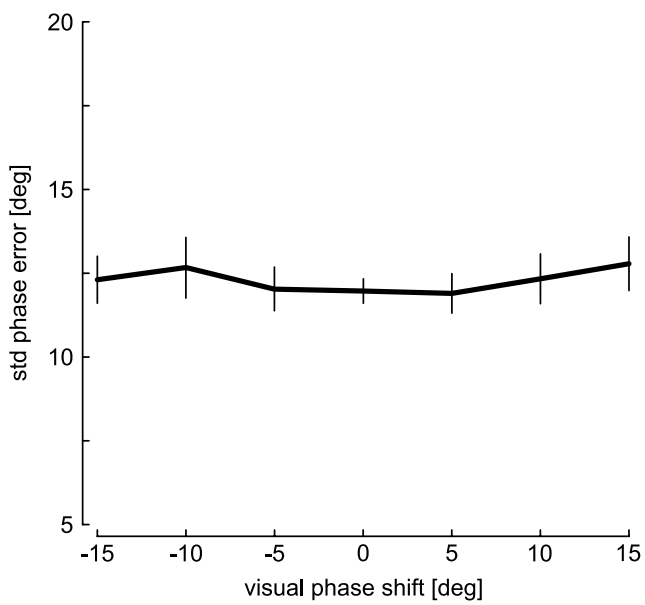

Figure 6. Average movement trajectory and phase difference when a phase shift is introduced in the visual display. $\boldsymbol{a}$, Average trajectories and corresponding visual display (AVF), for the six phase shifts introduced (from yellow to red) and without phase shift (black). All cycles — delimited by the maxima of the right wrist position — have been resampled by 360 equally time-spaced (he the averaged, and are displayed in the reference frame of the 1c). $\boldsymbol{b}$, Corresponding mean phase difference reights computed from Equation 8 and Figure $3 a$. The asterisks denote the phase shifts for which the measured compensation was not statistically different from this model-based prediction ( $t$ tests, all $p>0.05$ ). c, SD (std) of the same variable. The error bars in $\boldsymbol{b}$ and c represent the between-subjects SE.

ability (not adaptation). Burge et al. (2008) did not report whether the added random noise had an impact on the variability of the reaching endpoint.

The performers were not aware about the experimental manipulation, which aimed at testing how they adapted the stabilized pattern when the AVF was imperceptibly skewed (prediction 3). We confirmed here that the uncorrupted inputs and AVF (corrupted) were integrated to compute the perceived phase offset between both limbs. Qualitative examination of Figure $6 b$ suggests that the visual modality should account for $\sim 50 \%$ of the estimate, since the actual data slope was located exactly in between the two extremes: the "no-compensation" condition (if the shape of the visual display did not influence the stabilized pattern) and the "full-compensation" condition (if the movement was fully adapted to see a circle on the screen, whatever the phase shift). This result was quite consistent with the result from the first prediction, which weighted AVF at $\sim 50-60 \%$ (Fig. 4).

The partial compensation we observed in this experimental 
manipulation could also arise from the performers' inability to stabilize any arbitrary pattern: e.g., for a phase shift of $5^{\circ}$, full compensation required the production of the $85^{\circ}$-out-of-phase pattern, which could require extensive practice to be properly mastered. However, this explanation seems unlikely for the following two reasons. First, analyses of pattern variability across the different phase shifts did not expose any significant differences (Fig. 6c), suggesting that the required pattern was equally difficult to stabilize across all phase shifts. Second, the $85^{\circ}$-out-of-phase pattern was stabilized successfully, but only when the phase shift equaled $10^{\circ}$ rather than $5^{\circ}$ (Fig. $6 b$ ). So for this particular instance, the performers demonstrated that the pattern corresponding to full compensation at $5^{\circ}$ could be achieved, albeit in response to a different AVF phase shift. It seems reasonable to generalize this example to the whole range of coordination patterns covered by the experimental manipulation, since the data plotted in Figure $6 b$ are highly linear, and do not suggest any kind of saturation in the stabilized phase difference between both wrists.

The experimental manipulations revealed the ability to integrate two sensory modalities with some feedforward contribution. This third potential source of information could result from internal forward model computations (Wolpert et al., 1998; Kawato, 1999; Sabes, 2000), and was incorporated as a third sensory source in our model. The way we introduced this information source is an important issue, since feedforward- and feedbackdriven information are usually integrated with Kalman filters in the optimal control framework (Liu and Todorov, 2007). Predictions from Kalman filtering mainly deviate from those of MLE because motor noise sets both lower and upper bounds on the output variability, yet they reflect the same trend: the more sensory modalities, the more stable. Testing Kalman-based predictions in a design such as ours would be challenging, since it requires separating between motor and sensory noise, while-as we stated above-proprioceptive inflows cannot be entirely masked without highly invasive procedures. Moreover, our model fits are strong, suggesting that in this task, the difference between the two approaches would be small.

The ability to integrate different sensory modalities during cyclical movements has been observed along the whole learning process, since model-based prediction 1 and 2 were supported over the $5 \mathrm{~d}$ of practice (prediction 3 was not analyzed with the day of practice as design factor). The ability to rely on nearoptimal multisensory integration, even early on during skill learning, is generally consistent with previous investigations, demonstrating that the multisensory systems in adults are quite plastic across relatively short timescales (Ernst, 2007, 2008). A similar separation over two distinct timescales has recently been demonstrated in reaching movements within a stochastic curl field (Izawa et al., 2008): while the variability in the field induced rapid changes in the movement strategy, the overall optimization of reaching performance was only achieved after several days of practice. This last result is thus coherent with ours, demonstrating a separation between fast multisensory integrative capabilities, and slow learning processes.

Our contribution tackles a new area dealing with multisensory integration in the context of learning dynamic behaviors. We hypothesize that multisensory integration is achieved by mechanisms that are not task specific, and can be processed online approaching a statistically optimal manner, independent of the course of learning the task itself.

\section{References}

Alais D, Burr D (2004) The ventriloquist effect results from near-optimal bimodal integration. Curr Biol 14:257-262.

Bock O, Pipereit K, Mierau A (2007) A method to reversibly degrade proprioceptive feedback in research on human motor control. J Neurosci Methods 160:246-250.

Burge J, Ernst MO, Banks MS (2008) The statistical determinants of adaptation rate in human reaching. J Vis 8:20.1-20.19.

Casini L, Romaiguère P, Ducorps A, Schwartz D, Anton JL, Roll JP (2006) Cortical correlates of illusory hand movement perception in humans: a MEG study. Brain Res 1121:200-206.

Clark JJ, Yuille AL (1990) Data fusion for sensory information processing systems. Boston: Kluwer Academic.

Debaere F, Wenderoth N, Sunaert S, Van Hecke P, Swinnen SP (2003) Internal vs external generation of movements: differential neural pathways involved in bimanual coordination performed in the presence or absence of augmented visual feedback. Neuroimage 19:764-776.

Debaere F, Wenderoth N, Sunaert S, Van Hecke P, Swinnen SP (2004) Changes in brain activation during the acquisition of a new bimanual coordination task. Neuropsychologia 42:855-867.

Driver J, Noesselt T (2008) Multisensory interplay reveals crossmodal influences on 'sensory-specific' brain regions, neural responses, and judgments. Neuron 57:11-23.

Ernst MO (2007) Learning to integrate arbitrary signals from vision and touch. J Vis 7:7.1-7.14.

Ernst MO (2008) Multisensory integration: a late bloomer. Curr Biol 18:R519-R521.

Ernst MO, Banks MS (2002) Humans integrate visual and haptic information in a statistically optimal fashion. Nature 415:429-433.

Fisher NI (1983) Comment on a method for estimating the standard deviation of wind directions. J Appl Meteorol 22:1971.

Gilhodes JC, Roll JP, Tardy-Gervet MF (1986) Perceptual and motor effects of agonist-antagonist muscle vibration in man. Exp Brain Res 61:395-402.

Haken H, Kelso JA, Bunz H (1985) A theoretical model of phase transitions in human hand movements. Biol Cybern 51:347-356.

Helbig HB, Ernst MO (2007) Optimal integration of shape information from vision and touch. Exp Brain Res 179:595-606.

Hillis JM, Ernst MO, Banks MS, Landy MS (2002) Combining sensory information: mandatory fusion within, but not between, senses. Science 298:1627-1630

Hogan N, Sternad D (2007) On rhythmic and discrete movements: reflections, definitions and implications for motor control. Exp Brain Res 181:13-30.

Holmes NP, Spence C (2005) Multisensory integration: space, time and superadditivity. Curr Biol 15:R762-R764.

Izawa J, Rane T, Donchin O, Shadmehr R (2008) Motor adaptation as a process of reoptimization. J Neurosci 28:2883-2891.

Kawato M (1999) Internal models for motor control and trajectory planning. Curr Opin Neurobiol 9:718-727.

Kelso JAS (1995) Dynamic patterns. The self-organization of brain and behavior. Cambridge, MA: MIT.

Kersten D, Yuille A (2003) Bayesian models of object perception. Curr Opin Neurobiol 13:150-158

Körding KP, Wolpert DM (2004) Bayesian integration in sensorimotor learning. Nature 427:244-247.

Körding KP, Wolpert DM (2006) Bayesian decision theory in sensorimotor control. Trends Cogn Sci 10:319-326.

Körding KP, Wolpert DM (2007) Bayesian statistics and utility functions in sensorimotor control. In: Bayesian brain-probabilistic approaches to neural coding (Doya K, Ishii S, Pouget A, Rao RPN, eds). Cambridge, MA: MIT.

Körding KP, Beierholm U, Ma WJ, Quartz S, Tenenbaum JB, Shams L (2007) Causal inference in multisensory perception. PLoS ONE 2:e943.

Lee TD, Swinnen SP, Verschueren S (1995) Relative phase alterations during bimanual skill acquisition. J Mot Behav 27:263-274.

Liu D, Todorov E (2007) Evidence for the flexible sensorimotor strategies predicted by optimal feedback control. J Neurosci 27:9354-9368.

Macaluso E (2006) Multisensory processing in sensory-specific cortical areas. Neuroscientist 12:327-338.

Magill RA (2006) Motor learning and control: concepts and applications. McGraw-Hill, Ed 8. 
McCloskey DI, Cross MJ, Honner R, Potter EK (1983) Sensory effects of pulling or vibrating exposed tendons in man. Brain 106:21-37.

Roll JP, Vedel JP, Ribot E (1989) Alteration of proprioceptive messages induced by tendon vibration in man: a microneurographic study. Exp Brain Res 76:213-222.

Sabes PN (2000) The planning and control of reaching movements. Curr Opin Neurobiol 10:740-746.

Shams L, Seitz AR (2008) Benefits of multisensory learning. Trends Cogn Sci 12:411-417.

Sober SJ, Sabes PN (2003) Multisensory integration during motor planning. J Neurosci 23:6982-6992.

Sober SJ, Sabes PN (2005) Flexible strategies for sensory integration during motor planning. Nat Neurosci 8:490-497.

Stein BE, Stanford TR (2008) Multisensory integration: current issues from the perspective of the single neuron. Nat Rev Neurosci 9:255-266.

Swinnen SP (2002) Intermanual coordination: from behavioural principles to neural-network interactions. Nat Rev Neurosci 3:348-359.

Swinnen SP, Lee TD, Verschueren S, Serrien DJ, Bogaerds H (1997) Interlimb coordination: learning and transfer under different feedback conditions. Hum Mov Sci 16:749-785.
Tassinari H, Hudson TE, Landy MS (2006) Combining priors and noisy visual cues in a rapid pointing task. J Neurosci 26:10154-10163.

Trommershäuser J, Maloney LT, Landy MS (2008) Decision making, movement planning and statistical decision theory. Trends Cogn Sci 12:291-297.

van Beers RJ, Sittig AC, Denier van der Gon JJ (1999) Integration of proprioceptive and visual position-information: An experimentally supported model. J Neurophysiol 81:1355-1364.

Vaziri S, Diedrichsen J, Shadmehr R (2006) Why does the brain predict sensory consequences of oculomotor commands? Optimal integration of the predicted and the actual sensory feedback. J Neurosci 26:4188-4197.

Weerakkody NS, Mahns DA, Taylor JL, Gandevia SC (2007) Impairment of human proprioception by high-frequency cutaneous vibration. J Physiol 581:971-980.

Wolpert DM, Miall RC, Kawato M (1998) Internal models in the cerebellum. Trends Cogn Sci 2:338-347.

Zanone PG, Kelso JA (1992) Evolution of behavioral attractors with learning: nonequilibrium phase transitions. J Exp Psychol Hum Percept Perform 18:403-421. 